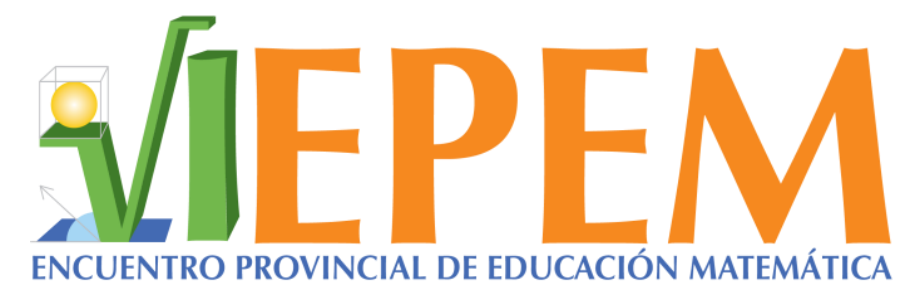

VI Encuentro Provincial de Educación Matemática.

27 al 29 de setiembre, 2017. Puntarenas, Costa Rica.

\title{
Actitud del docente de educación secundaria hacia la historia de las matemáticas: validación de un instrumento de recolección de información.
}

\author{
Adriana Álvarez Carranza \\ adri104_@hotmail.com \\ Estudiante \\ Universidad Nacional \\ Costa Rica \\ Leonel David Navarro Castillo \\ 1dnavarroc@hotmail.com \\ Estudiante \\ Universidad Nacional \\ Costa Rica \\ Ana Lucía Alfaro Arce \\ aalfar@una.cr \\ Universidad Nacional \\ Costa Rica
}

\begin{abstract}
Resumen
Esta ponencia corresponde a un avance de investigación, se presentan los resultados de un estudio piloto que tiene como fin determinar la confiabilidad y validación de un cuestionario construido para describir la actitud, la formación y el uso de la historia de las matemáticas manifestadas por los docentes de educación secundaria, en Costa Rica. Los componentes cognitivo, afectivo y comportamental que conforman una actitud, permitirán establecer la actitud del docente hacia la historia de las matemáticas, que será clasificada en positiva o negativa. En este avance se describe la experiencia de los investigadores en el proceso de validación del instrumento y como resultados principales de la prueba piloto se destacan un Alfa de Cronbach de 0,931 y una actitud positiva hacia la historia de las matemáticas por parte del profesorado.

Palabras clave: Historia de la matemática; actitud del docente; educación secundaria, validación y confiabilidad.
\end{abstract}

Ponencia

Álvarez, A.; Navarro, L. y Alfaro, A. (2017). Actitud del docente de educación secundaria hacia la historia de las matemáticas: validación de un instrumento de recolección de información. En Y.

Morales-López, M. Picado, R. Gamboa, C. Martínez, M. Castillo y R. Hidalgo (Eds.), Memorias del VI Encuentro Provincial de Educación Matemática, Costa Rica, 2017 (pp. 4-12). Heredia:

Universidad Nacional. ISBN: 978-9968-9661-5-3. DOI: http://dx.doi.org/10.15359/epem.6.2 


\section{Introducción}

Los programas de estudio en Matemática del Ministerio de Educación Pública (MEP) (2012) establecen cinco ejes disciplinares específicos a saber:

La resolución de problemas como estrategia metodológica principal, la contextualización activa como un componente pedagógico especial, el uso inteligente de tecnologías digitales, la potenciación de actitudes y creencias positivas en torno a las Matemáticas y el uso de la historia de las Matemáticas (p.11).

Con respecto a la historia de las Matemáticas su selección se debe a que "ha estado prácticamente ausente de los programas y de la práctica educativa" (Ruiz, 2012, p.48). Su papel en la enseñanza secundaria del país se debe concebir como "un recurso que proporcione oportunidades didácticas especiales, que le ofrezca insumos a la lógica de la lección y genere actitudes y creencias positivas sobre las matemáticas" (MEP, 2012, p.64). Pese a que el MEP propone la incorporación de este eje en la enseñanza, esto no garantiza que en la práctica docente se esté abordando como tal. La actitud (positiva o negativa) del profesorado hacia las exigencias curriculares del sistema, en relación con este eje, podría reflejarse en su manera de abordarlo en la enseñanza. De ahí el interés por saber, ¿Cuál es la actitud hacia la historia de la matemática que manifiestan algunos docentes de educación secundaria?

El objetivo general del estudio piloto es describir la actitud, la formación y el uso de la historia de las matemáticas como recurso didáctico que manifiestan los docentes de educación secundaria de las regiones educativas Heredia y San José Central.

\section{Marco teórico}

Para efectos del estudio se definió actitud como la predisposición del docente de matemáticas a reaccionar de manera valorativa (positiva o negativa) hacia un objeto o una situación dada, susceptible al cambio y reflejada en el comportamiento a seguir. Asimismo, se entenderá la actitud hacia la historia de las matemáticas, como una predisposición positiva o negativa del docente, en función de los conocimientos históricos (teóricos y metodológicos) de las matemáticas.

Cabe mencionar que la actitud de una persona hacia determinada situación o acontecimiento se puede determinar mediante aquellos aspectos cognitivos, afectivos y conductuales que exprese el individuo. Para este estudio, se adoptarán las siguientes definiciones dadas por Castro de Bustamante (2003) con las que se construyó una escala de actitud tipo Likert en el cuestionario elaborado para la investigación.

Comportamiento cognitivo: incluye hechos, opiniones, creencias, pensamientos, valores, conocimientos y expectativas (especialmente de carácter evaluativo) acerca del objeto de la actitud.

Comportamiento afectivo: son los procesos que avalan o contradicen las bases de nuestras creencias, expresados en sentimientos evaluativos y 
preferencias, estados de ánimo y las emociones que se evidencian (física y/o emocionalmente) ante el objeto de la actitud (tenso, ansioso, feliz, preocupado, dedicado, apenado).

Comportamiento comportamental: muestran las evidencias de actuación a favor o en contra del objeto o situación de la actitud, amén de la ambigüedad de la relación "conducta-actitud" (p.4).

Es necesario que cada ítem y pregunta que conforma la escala y el cuestionario sean pertinente en la investigación, por tal razón, se consideran aspectos de confiabilidad, validez y objetividad en los mismos.

Según Hernández, Fernández y Baptista (2010) la confiabilidad "se refiera al grado en que un instrumento produce resultados consistentes y coherentes” (p.200). Estos mismos autores se refieren a la validez en términos generales como "al grado en que un instrumento realmente mide la variable que se pretende medir" (p.201), además, expresan que en la validez pueden existir distintos tipos de evidencia, como, la evidencia de contenido, criterio y constructo.

En este caso, se comentará la validez del contenido y constructo, con respecto a la primera, según Hernández, Fernández y Baptista (2010) "se refiere al grado en que un instrumento refleja un dominio específico de contenido de lo que se mide" (p.201); es decir, que cada aspecto representado en el instrumento debe tener relación directa con los objetivos de la investigación, evitando incluir aspectos sobrantes o por el contrario excluir aquellos que son pertinentes para el estudio.

Leyva (2011) menciona que una de las aproximaciones generales utilizadas para establecer la validez de contenido de una prueba referida a criterio, es "involucrar los juicios emitidos por especialistas en el contenido" (p.147), por tal razón, dicha aproximación será de gran importancia y se describirá en el marco metodológico de esta investigación.

Con respecto a la validez de constructo para Grinnell, Williams y Unrau (2009) es probablemente la más importante, principalmente desde la perspectiva científica y se refiere a qué tan exitosamente un instrumento representa y mide un concepto teórico (citado por Hernández, Fernández y Baptista, 2010, p.203). En esta investigación, la actitud representa un constructo, el cual es posible expresar teóricamente en conjunto a través de los comportamientos cognitivos, afectivos y conductuales.

Por otra parte, según Mertens (2005) la objetividad "se refiere al grado en que el instrumento es permeable a la influencia de los sesgos y tendencias del investigador o investigadores que lo administran, califican e interpretan" (citado por Hernández, Fernández y Baptista, 2010, p.203), es decir, consiste en la transparencia del instrumento, evitando en lo posible que la subjetividad de quienes participan en su elaboración y validación interfiera de cierto modo en los resultados.

En el instrumento elaborado se incorporan aspectos sobre los usos de la historia de la matemática, el MEP (2012) recomienda como opciones didácticas:

reservatorio de anécdotas para motivar y sensibilizar, descripción de situaciones que sitúen contextos y circunstancias socioculturales, explicación de la secuencia o lógica del desarrollo de los contenidos matemáticos y como una fuente primaria al presentarle a los estudiantes, 
algunos tópicos o problemas con las herramientas teóricas que se disponían en un momento histórico (p.65).

De igual manera, al investigar sobre los usos que otros autores le dan a la historia de la matemática, estos en ocasiones coinciden con los propuestos por el MEP por tal razón, en la elaboración del cuestionario se consideran dichos usos.

\section{Metodología}

Se sigue un enfoque cuantitativo, de tipo descriptivo, ya que se persigue "especificar las propiedades, las características y los perfiles de personas, grupos, comunidades, procesos, objetos o cualquier otro fenómeno que se someta a un análisis" (Hernández, Fernández y Baptista, 2010, p.80).

En una prueba piloto, se recomienda elegir "un pequeño grupo de sujetos que no pertenezcan a la muestra seleccionada pero sí a la población o un grupo con características similares a la de la muestra del estudio, aproximadamente entre 14 y 30 personas" (Corral, 2009, p.238). Por esta razón, se seleccionaron 20 docentes de colegios públicos y subvencionados, de dos Direcciones Regionales de Educación del país, que no eran parte de la muestra, pero que pertenecen a la población del estudio que engloba dicha prueba. En la tabla adjunta, se muestra la selección:

Tabla 1

Cantidad de docentes de matemáticas en cada Dirección Regional Educativa por colegio público y subvencionado para la prueba piloto, según datos del año 2016

\begin{tabular}{llc}
\hline Región & Colegio & Cantidad \\
\hline \multirow{2}{*}{ San José Central } & Unidad Pedagógica Colegio República de México & 5 \\
& Liceo del Sur & 5 \\
\hline \multirow{2}{*}{ Heredia } & C.T.P Mercedes Norte & 3 \\
& C.T.P San Pedro de Barva & 3 \\
\hline Total & C.T.P de Ulloa & 4 \\
\hline
\end{tabular}

Fuente: Elaboración propia con información del Departamento de Análisis Estadístico, Dirección de Planificación Institucional del Ministerio de Educación Pública.

Por tratarse de un estudio enfocado en la descripción de la actitud, se utilizará como instrumento un cuestionario. Para la construcción del mismo se siguieron varias etapas basadas en el marco teórico de la investigación. Contiene preguntas para las variables de interés en el estudio, interrogantes relacionadas a la formación en historia de las matemáticas y sus usos, así como una escala de Likert. En este último punto, se presentan treinta proposiciones (diez para cada componente) referidas a determinar la actitud de los docentes hacia la historia de las matemáticas y cada planteamiento es calificado con cinco categorías de respuesta para elegir: siempre, casi siempre, algunas veces, muy pocas veces y nunca. La validez del contenido y del constructo actitud, se realizó mediante el juicio de expertos, docentes afines al área de estadística e historia de las matemáticas, también con algunos académicos de la Escuela de Matemática de la Universidad Nacional. Para esta etapa se elaboró un instrumento propio de validación para el cuestionario, en el cual se consideran aspectos como: redacción adecuada, pertinencia de las preguntas e ítems con el 
objetivo correspondiente, pertinencia de cada ítem con su componente de la actitud, orden y pases adecuados en las preguntas y otras recomendaciones.

La confiabilidad se midió mediante el alfa de Cronbach en la prueba piloto, siguiendo las recomendaciones dadas por Hernández, Fernández y Baptista (2010) para el grado de correlación "si se obtiene 0,25 en la correlación o coeficiente, esto indica baja confiabilidad; si el resultado es 0,50 , la fiabilidad es media o regular. En cambio, si supera el 0,75 es aceptable, y si es mayor a 0,90 es elevada" (p.302).

A partir de los datos obtenidos en la prueba piloto se realizó el cálculo del índice de discriminación con la versión de prueba del software Statistical Package for the Social Sciences (SPSS 20). Se siguen las recomendaciones de Lozano y de la Fuente (2015), sintetizadas en la siguiente tabla, con el fin de decidir si algunos de los ítems en la escala de actitud serán excluidos en el instrumento.

Tabla 2

Interpretación del índice de discriminación.

\begin{tabular}{ll}
\hline \multicolumn{1}{c}{ Valores } & \multicolumn{1}{c}{ Interpretación } \\
\hline Igual o mayor que 0,40 & El ítem discrimina muy bien. \\
\hline Entre 0,30 y 0,39 & El ítem discrimina bien. \\
\hline Entre 0,20 y 0,29 & El Ítem discrimina poco. \\
\hline Entre 0,10 y 0,19 & Ítem límite. Se debe mejorar. \\
\hline Menor de 0,1 & El ítem carece de utilidad para discriminar. \\
\hline
\end{tabular}

Fuente: Lozano y de la Fuente (2015, p.259)

Las proposiciones que se mantendrán para el cuestionario final serán aquellos ítems que tengan un índice de discriminación mayor o igual a 0,3.

Cabe mencionar que, en este estudio, se ha definido que el docente manifestará una actitud positiva en el caso en que obtenga un puntaje entre 90 y 150, incluyendo los valores extremos, por el contrario, manifestará una actitud negativa en el caso contrario. La siguiente figura representa la escala de decisión para definir una actitud positiva o negativa del docente.

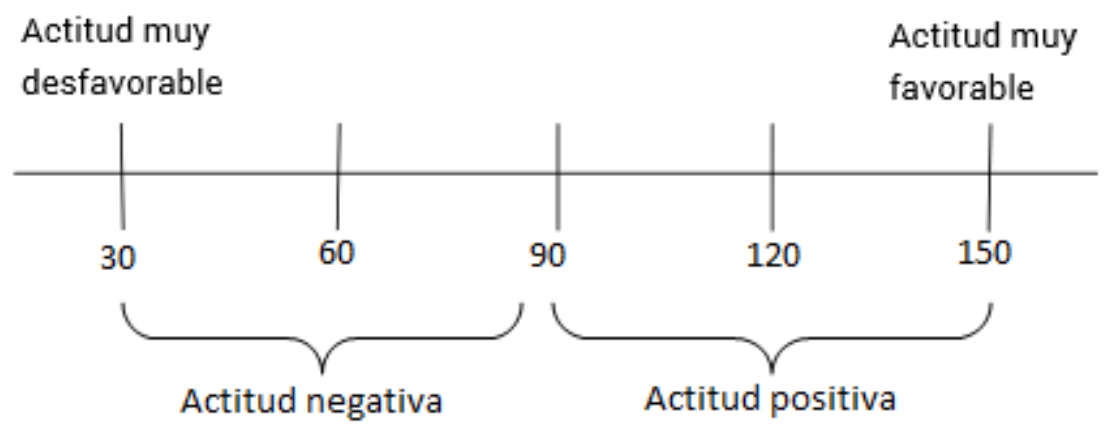

Figura 1. Clasificación de la actitud (positiva o negativa) del docente. 


\section{Análisis}

Para el análisis de la información recolectada se construyó una base de datos en el software Statistical Package for the Social Sciences (SPSS 20). De los 20 docentes pertenecientes a los colegios seleccionados para la prueba piloto, únicamente 17 accedieron a completar el cuestionario y con el fin de aumentar el número de participantes, se le solicitó completar el cuestionario a 11 docentes inscritos como tutores en la Actividad Académica Matemática para la Enseñanza Media (MATEM) de la Escuela de Matemática de la Universidad Nacional, debido a que los mismos cuentan con características similares a la población de estudio, al final se aplicó a un total de 28 docentes de matemáticas de educación secundaria. Dado que hubo 3 casos de cuestionarios incompletos, para efectos del cálculo del Alfa de Cronbach estos fueron excluidos.

Tabla 3

Resumen del procesamiento de los casos

\begin{tabular}{ccc}
\hline Casos & $\mathrm{N}^{\circ}$ de Casos & $\%$ \\
Válidos & 25 & 89.3 \\
Excluidos a. & 3 & 10.7 \\
\hline Total & 28 & 100.0 \\
\hline
\end{tabular}

a. Eliminación por lista basada en todas las variables del procedimiento.

Fuente: Elaboración propia

Considerando la información anterior, el Alfa de Cronbach tiene un valor de 0,931 lo cual establece que la correlación es elevada. Con respecto al índice de discriminación para cada ítem, se tiene la siguiente información:

Tabla 4

Cálculo del índice de discriminación

\begin{tabular}{lcc}
\hline \multicolumn{1}{c}{ Ítems } & $\begin{array}{c}\text { Índice de } \\
\text { discriminación }\end{array}$ & $\begin{array}{c}\text { Alfa de Cronbach si se } \\
\text { elimina el elemento }\end{array}$ \\
\hline $\begin{array}{l}\text { A.1 Me desmotiva involucrar la historia de las } \\
\text { Matemáticas en el planeamiento de las lecciones. }\end{array}$ & .269 & .932 \\
$\begin{array}{l}\text { A.2 Me siento presionado(a) por el MEP a } \\
\text { incorporar la historia de las Matemáticas en las } \\
\text { lecciones. }\end{array}$ & .418 & .930 \\
$\begin{array}{l}\text { A.3 Disfruto resolver problemas que involucran } \\
\text { aspectos de la historia de las Matemáticas durante }\end{array}$ & .610 & .928 \\
$\begin{array}{l}\text { las lecciones. } \\
\begin{array}{l}\text { A.4 Prefiero no usar aspectos históricos de las } \\
\text { matemáticas al enseñar esta disciplina. }\end{array}\end{array}$ \\
$\begin{array}{l}\text { A.5 Me siento inseguro(a) al utilizar la historia de } \\
\text { las Matemáticas al desarrollar actividades didácticas } \\
\text { durante las lecciones. }\end{array}$ \\
$\begin{array}{l}\text { A.6 Me siento intimidado(a) al enfrentarme a la } \\
\text { resolución de problemas que involucren aspectos } \\
\text { históricos de las Matemáticas. }\end{array}$ \\
$\begin{array}{l}\text { A.7 Me aburre capacitarme en temas relacionados } \\
\text { con la historia de las Matemáticas y su }\end{array}$ \\
implementación en las lecciones.
\end{tabular}


A.8 Me agobia dedicar tiempo para leer aspectos históricos de las Matemáticas para poder

implementarla en las lecciones.

A.9 Me angustia utilizar la historia de las

Matemáticas en el desarrollo de las lecciones, por el

tiempo que se invierte.

A.10 Me preocupa que la historia de las

Matemáticas alcance un papel primordial en los

programas de estudio de matemáticas.

B.1 Considero fácil la implementación de la historia de las Matemáticas como recurso didáctico en las

lecciones.

B.2 El uso de la historia de las Matemáticas permite una comprensión profunda de las Matemáticas.

B.3 Considero importante usar la historia de las Matemáticas en el desarrollo de las lecciones.

B.4 Considero que los conocimientos sobre historia de las Matemáticas son secundarios en la formación de todo docente de matemáticas.

B.5 Los conocimientos históricos de las matemáticas que adquieren los estudiantes de secundaria, los motiva en su aprendizaje.

B.6 La historia de las Matemáticas complementa los contenidos por desarrollar en las lecciones.

B.7 La historia de las Matemáticas proporciona herramientas didácticas para el desarrollo de los contenidos.

B.8 Incorporar la historia de las Matemáticas en el planeamiento de las lecciones demanda mucho tiempo.

B.9 Considero que la formación que recibí en historia de las Matemáticas es insuficiente para utilizarla en mis lecciones.

B.10 Considero que con el uso de la historia de las Matemáticas mi labor docente no mejora.

C.1 Utilizo la historia de las Matemáticas como un medio didáctico para el desarrollo de mis lecciones.

C.2 Utilizo la historia de las Matemáticas en las lecciones a través de anécdotas de personajes matemáticos.

C.3 Utilizo la historia de las Matemáticas en las lecciones para introducir un contenido.

C.4 Implemento la historia de las Matemáticas en las lecciones para describir situaciones sociales o

C.5 Procuro incorporar la historia de las

Matemáticas en algún tipo de evaluación formativa.

C.6 La historia de las Matemáticas me permite fundamentar los contenidos matemáticos. 
C.7 Utilizo la historia de las Matemáticas para desarrollar los contenidos matemáticos según el

.734

orden histórico cronológico.

C.8 Utilizo la historia de las Matemáticas al elaborar problemas matemáticos para mis estudiantes.

C.9 Evito motivar el gusto hacia la historia de las

Matemáticas en las demás personas.

C.10 Utilizo situaciones propias de la historia de las Matemáticas para reforzar valores en los estudiantes.

Fuente: Elaboración propia

En caso de eliminar los ítems A.1 B.4 y C.9 siendo estos los que presentan un índice de discriminación menor a 0,3, el alfa de Cronbach sería de 0,951. No obstante, por la naturaleza descriptiva de la investigación y debido a que dichos ítems recogen información importante a valorar sobre aspectos de la historia de la matemática, para efectos de la versión final del cuestionario se tomaran en cuenta.

Con respeto a la actitud, se obtuvo que la mayoría de los docentes muestran una actitud positiva hacia la historia de las matemáticas.

\section{Conclusiones}

Aprender a realizar una prueba piloto en una investigación es fundamental. Se requiere una planificación cuidadosa y responsable, ya que los resultados finales serán válidos y confiables dependiendo de esta. Los resultados del presente estudio piloto han permitido determinar la confiabilidad (Alfa de Cronbach con un valor de 0,931) y validez del cuestionario diseñado para describir la actitud, la formación y el uso de la historia de las matemáticas manifestadas por los docentes de educación secundaria, en Costa Rica.

Además fue posible determinar que la actitud que manifiestan algunos docentes de educación secundaria hacia la historia de las matemáticas es favorable o positiva, así como la formación que han recibido en esta área y los usos que le dan en educación secundaria.

Este estudio resultaría un aporte para la comunidad de investigadores en el campo de la matemática educativa, al ofrecer una guía, del procedimiento de validación de un cuestionario, que sirva de apoyo en la elaboración de futuras investigaciones afines a la actitud hacia la historia de la matemática.

\section{Referencias}

Castro de Bustamante, J. (2003). Análisis de los Componentes actitudinales de los docentes hacia la enseñanza de la Matemática. Caso: $1^{a}$ y $2^{a}$. (Tesis doctoral). Universitat Rovira I Virgili. Cataluña, España.

Hernández, R., Fernández, C. y Baptista, P. (2010). Metodología de la Investigación (5ta Ed). México: McGraw-Hill/ Interamericana editores, S.A.DE C.V.

Leyva, Y. (enero, 2011). Una reseña sobre la validez de constructo de pruebas referidas a criterio. Perfiles educativos, 33(131), 131-154.

Lozano, L. y de la Fuente, E. (2015). Diseño y validación de cuestionarios. En Pantoja, A. (coordinador), Manual básico para la realización de tesinas, tesis y trabajos de investigación. España: Editorial EOS (pp. 249-272) 
Ministerio de Educación Pública. (2012). Reforma Curricular en Ética, Estética y Ciudadanía. PROGRAMAS DE ESTUDIO DE MATEMÁTICAS, I, II y III Ciclos de la Educación General Básica y Ciclo Diversificado. San José, Costa Rica: Autor.

Ruíz, A. (2012). Curso bimodal para el Ciclo Diversificado: Enfoque de Resolución de problemas. Recuperado de http://www.reformamatematica.net/programas/index.php/documentosbimodales/articl e/view/47/86

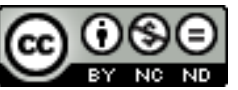

Esta obra está bajo una licencia de Creative Commons Reconocimiento-NoComercialSinObraDerivada 4.0 Internacional. 